\title{
Simulation of Magnetorheological Fluids Based on Lattice Boltzmann Method with Double Meshes
}

\author{
Xinhua Liu, Hao Liu, and Yongzhi Liu \\ School of Mechanical and Electrical Engineering, China University of Mining and Technology, \\ Xuzhou 221116, China \\ Correspondence should be addressed to Xinhua Liu, 1_xinhua_2006@126.com
}

Received 16 May 2012; Accepted 27 July 2012

Academic Editor: Subhas Abel

Copyright (C 2012 Xinhua Liu et al. This is an open access article distributed under the Creative Commons Attribution License, which permits unrestricted use, distribution, and reproduction in any medium, provided the original work is properly cited.

\begin{abstract}
In order to study the rheological characteristics of magnetorheological fluids, a novel approach based on the two-component Lattice Boltzmann method with double meshes was proposed, and the micro-scale structures of magnetorheological fluids in different strength magnetic fields were simulated. The framework composed of three steps for the simulation of magnetorheological fluids was addressed, and the double meshes method was elaborated. Moreover, the various internal and external forces acting on the magnetic particles were analyzed and calculated. The two-component Lattice Boltzmann model was set up, and the flowchart for the simulation of magnetorheological fluids based on the two-component Lattice Boltzmann method with double meshes was designed. Finally, a physics experiment was carried out, and the simulation examples were provided. The comparison results indicated that the proposed approach was feasible, efficient, and outperforming others.
\end{abstract}

\section{Introduction}

Magnetorheological fluids (MRFs) are a kind of colloidal suspension well-dispersing micronic magnetic particles with a size of about $10 \mathrm{~nm}$ in diameter in a carrier liquid. As a type of novel functional material, MRFs have a bright application future in many fields such as bioengineering mechanical, engineering, aerospace, and medical science. The rheological characteristics of magnetorheological fluids can be controlled by an applied magnetic field, and the theoretical analysis and experimental results have shown that the catenulate microstructure of magnetorheological fluids is the most important factor for the magnetorheological effect [1]. Thus, it is essential and important to make a detailed research into the micromechanism of the magnetorheological effect.

Nowadays, the numerical simulation method has been applied as an important and effective solution for computational fluid dynamics, but it is difficult for the conventional 
numerical simulation methods to simulate complicated magnetorheological fluids. It can not reveal the various interactions between microcosmic particles by macroscopic methods such as finite-element method, finite-difference method, and finite-volume method. With microscopic methods, it can only simulate a small number of particles because of vast calculating amount. Compared with conventional numerical simulation methods, the Lattice Boltzmann method (LBM) has many advantages such as high amenability to parallel computing, easy dealing with the complex boundary conditions, the physical representation of microscopic interactions and easy implementing program, and so forth. All these advantages make LBM gradually becomes a popular method for the simulation of a range of complex fluids. During the simulation of complex fluids, it follows a prescribed Lattice Boltzmann evolution equation instead of the macroscopical Navier-Stokes equations, and it has been confirmed that the Navier-Stokes equations can be recovered from the Boltzmann equation by Chapman-Enskog expansion.

As a widely applied model, the standard Lattice Boltzmann model is developed from the Lattice Gas Cellular Automata (LGCA). Thus, the meshes must be consistent with the discrete-velocity to ensure that particles can migrate to the adjacent nodes at the next time step, and the standard Lattice Boltzmann model has the advantages of simple principle, simple operation and high amenability to parallel computing, and so forth. However, along with the applications and developments of Lattice Boltzmann model in many fields, a great limitation has been demonstrated in simulation process with standard Lattice Boltzmann model. On the one hand, the applications are limited because the whole flow field must be divided into symmetrical meshes such as quadrangle and hexagon, and so forth. On the other hand, because of the determinate time accuracy, calculation precision cannot be altered according to the actual research question. In order to overcome the existing defects and enlarge the application fields, many scholars were devoted to the relevant research and presented some improvement methods. In [2], He et al. presented an interpolationsupplemented Lattice Boltzmann method through introducing interpolation into Lattice Boltzmann method. Taylor series expansion and least square-based Lattice Boltzmann method based on the least square optimization which was first presented by Shu et al. [3]. In [4], Filippova and Hänel applied the idea of multiblock grid technique to the Lattice Boltzmann method, and further theoretical study was carried out by Allen [5]. In [6], Tölke et al. applied multigrid method to the Lattice Boltzmann equation. Double meshes method is a very effective approach in improving convergence rate of algebraic equations. Classical iterative algorithms can efficiently decrease the error at high frequency, but it is difficult for low frequency. Through double meshes method, iteration process makes the high frequency error reduce on the fine mesh, and on the coarse mesh, iteration process can decrease the low frequency error. Although the attenuation velocity of low frequency error is slow on the coarse mesh, convergence rate is quick due to a small quantity of calculating amount [7].

Bearing the above observations in mind, we present a two-component Lattice Boltzmann method with double meshes to simulate magnetorheological fluids in an external uniform magnetic field and the rest of this paper is organized as follows. In Section 2 some related works are outlined based on literature. Section 3 presents the double meshes method and two-component Lattice Boltzmann model, and the stress state of magnetic particles in an external magnetic field is analyzed and calculated. Section 4 gives the detailed process for the simulation of magnetorheological fluids based on Lattice Boltzmann method with double meshes. Section 5 illustrates the simulation results of magnetorheological fluids and compares them with the physics experimental results and other methods. Finally, Section 6 concludes with some advantages of the two-component Lattice Boltzmann method with 
double meshes applied to the simulation of magnetorheological fluids and points out some future work.

\section{Literature Review}

\subsection{The Computer Simulation Methods of Magnetorheological Fluids}

In order to research the character of magnetorheological fluids, many scholars have worked on the problem and proposed some different solutions. Monte Carlo method has been well accepted as a useful approach to simulate magnetorheological fluids. In [8], Peng et al. investigated and simulated a suspension comprised of magnetic and nonmagnetic particles in gradient magnetic fields using a two-dimensional Monte Carlo simulation. Moreover, Lattice Boltzmann method has gradually been applied for the simulation of magnetorheological fluids. In [9], Xuan et al. developed a Lattice Boltzmann model to simulate the distribution of suspended magnetic nanoparticles and morphology of the ferrofluid. Moreover, many other methods are adopted to simulate magnetorheological fluids. In [10], Haitao and Xianghe adopted molecular dynamics simulation method to simulate the catenulate structure of magnetorheological fluids under the action of an external magnetic field. In [11], Li et al. presented three dimensions dynamic models of magnetic particles based on the theory of magnetic dipoles to simulate the procession of magnetorheological fluids magnetic particles forming chains in an external magnetic field. In [12], Ly et al. employed the boundary element method (BEM) to carry out dynamic simulation of magnetorheological fluids. In [13], Zhang et al. divided magnetorheological fluids into two coincident elements to simulate the mechanical behavior of magnetorheological fluids subjected to magnetic field in the preyield region by the finite element analysis software ANSYS.

\subsection{The Applications of Lattice Boltzmann Method}

Although the Lattice Boltzmann method was introduced only 20 years, it has been widely applied to simulate a wide range of physical phenomena. In [14], Zhou et al. presented a novel hybrid method based on the Lattice Boltzmann approach to get insight into the micro-scale characteristics of the multicomponent flow of nanofluid. In [15-17], Lattice Boltzmann model was successfully applied to simulate flow and heat transfer in a porous medium of complex structure. In [18], Heuveline et al. applied a hybrid parallelization of Lattice Boltzmann method to a 3D benchmark problem and compared with a purely MPI based approach. The Lattice Boltzmann models have also been applied to solve nonlinear partial differential equations such as Korteweg-de Vries equation [19], Poisson' equation [20], nonlinear convection-diffusion equations [21], and Burgers' equation [22]. In [23], Hyakutake et al. investigated the blood cell behavior in microvascular flows by Lattice Boltzmann simulations of a particulate suspension in a Poiseuille flow and bifurcation flow.

\subsection{Discussion}

Although many approaches for the simulation of magnetorheological fluids have been developed in above literature, they have some common disadvantages described as follows. Firstly, for the complex fluids such as magnetorheological fluids, they cannot get accurate simulation result compared with the real physics experiment. Secondly, it would take a lot of time in the simulation of magnetorheological fluids with mass volume fraction. Finally, 


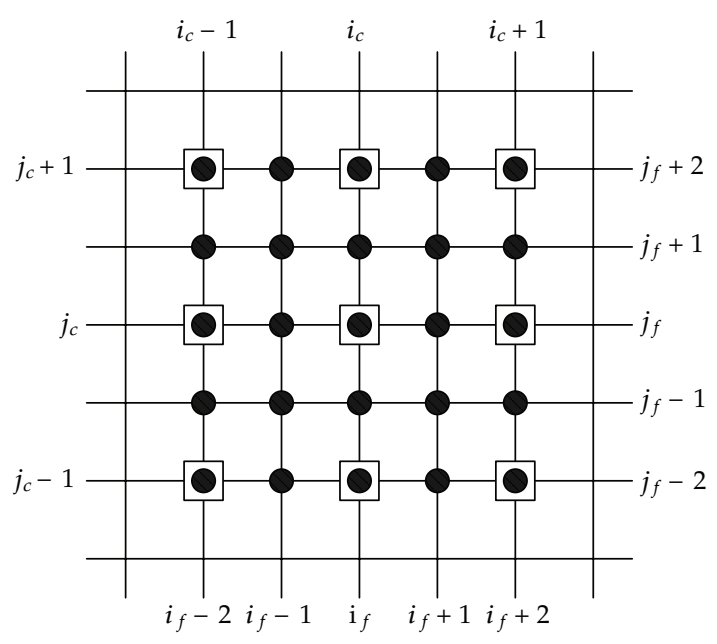

Figure 1: Structure of the double meshes.

it is very difficult to simulate fluids with complex boundary conditions. Based on our past research on the computer simulation of magnetorheological fluids and Lattice Boltzmann method, this paper tries to tackle the above problems.

In this research, a two-component Lattice Boltzmann method with double meshes is proposed for the simulation of magnetorheological fluids to overcome above disadvantages.

\section{The Two-Component Lattice Boltzmann Method with Double Meshes}

The framework for the simulation of magnetorheological fluids is composed of three steps described as follows. Firstly, two different-sized uniform meshes are constructed on the whole magnetorheological fluids, and the spatial step of the coarse mesh is generally double-size that of the fine mesh. Secondly, the various internal and external forces acting on the magnetic particles are analyzed and calculated. Finally, the two-component Lattice Boltzmann model is proposed and applied to the simulation of magnetorheological fluids on the two different-sized uniform meshes with taking the above forces into account.

\subsection{Double Meshes Method}

The structure of double meshes is shown as Figure 1, where two different-sized uniform meshes are employed for the simulation of magnetorheological fluids. The subscript $c$ and $f$, respectively, denote the coarse mesh and fine mesh, and the spatial step of the coarse mesh is generally double-size that of the fine mesh.

It iterates and evolves on two different scale meshes by the two-component Lattice Boltzmann method with double meshes and the relaxation process decreases the high frequency error on the fine mesh and the low frequency error on the coarse mesh, respectively.

During the simulation of magnetorheological fluids in a uniform magnetic field, a preliminary simulation result is obtained after iterating for $m$ times on the fine mesh and then transfers it to the coarse mesh. After that, a more precise result is obtained after iterating for $n$ times on the coarse mesh and then transfers it to the fine mesh. The deviation of simulation 
results can be decreased gradually and a quite precise simulation result can be achieved through the above iteration process [24].

\subsection{Force Analysis of Magnetic Particles}

In a multiphase magnetorheological fluids system, the character of magnetorheological fluids is mainly determined by the various internal and external forces acting on magnetic particles. In presence of an external uniform magnetic field, magnetic particles are mainly affected by the brown force, viscous resistance, magnetic force, van der Waals force, repulsive force, gravitational force and buoyancy, and so forth.

\subsubsection{Brownian Force}

The Brownian force results from random thermal motion of the magnetic particles and impact with others suspended in the carrier liquid. Due to the noncorrelation of the forces at different time, the Brownian force can be modeled as the mean for zero of a Gaussian white noise process [25]. The Brown force can be expressed as follows:

$$
F_{i}^{B}=\varsigma \cdot \sqrt{\frac{12 \pi a \mu k_{B} T}{\Delta t}}
$$

where $\varsigma$ is the Gaussian random number with a mean value of zero and unit variance, $k_{B}$ is the Boltzmann constant, $T$ is the absolute temperature, and $\Delta t$ is the magnitude of the time step.

\subsubsection{Viscous Resistance}

It is assumed that the carrier liquid is incompressible and micronic magnetic particles are spherical, and the viscous resistances of magnetic particles can be described by Stokes formula as follows [26]:

$$
F_{i}^{V}=-3 \pi \eta d \Delta u
$$

where $\Delta u$ is the velocity difference between magnetic particles and carrier liquid, $\eta$ is the kinematic viscosity, and $d$ is the particle diameter of magnetic particles.

\subsubsection{Magnetic Force}

In our paper, the uniform magnetic field is applied to study the character of magnetorheological fluids and each magnetic particle is affected by a force because of the dipolar interactions among the magnetic particles.

As a single-domain granule, each magnetic particle has its intrinsic magnetic moment whose size and direction are unceasingly changing according to the change of the external magnetic field and its own movement. The particles interaction force can be formulated by calculating the interactive forces between two magnetic dipoles. 
Magnetic moment of magnetic particles in a magnetic field can be calculated as follows:

$$
m=\frac{4}{3} \pi R^{3} X H
$$

where $R$ is the radium of magnetic particles, $X$ is the magnetic susceptibility, and $H$ is the strength of external magnetic field.

According to the theory of magnetic dipole, the magnetic force acting on one magnetic particle from others can be expressed as follows [27]:

$$
F_{i}^{M}=\sum_{j \neq i} \frac{3 u_{0}}{4 \pi r_{i j}{ }^{4}}\left[\left(m_{i} \cdot m_{j}\right)-3\left(m_{i} \cdot r_{0}\right)\left(m_{j} \cdot r_{0}\right)\right]
$$

where $r_{i j}$ is the relative position vectors between two magnetic particles and $r_{0}$ is the unit vector of $r_{i j}$.

\subsubsection{Van Der Waals Force}

The van der Waals force is a kind of ubiquitous interactive force which is not inherent in magnetorheological fluids. It is a short range interactive force, and it will decline rapidly with the increasing of distance between the molecules. The van der Waals force from other spheres separated by a distance $h$ can be calculated by Hamaker's formula as follows [28]:

$$
F_{i}^{W}=\sum_{j \neq i}-\frac{A(16 \lambda)^{3}}{3\left(R_{i}+R_{j}\right)} \times\left\{\frac{s}{(1+\lambda)^{2}\left[s^{2}(1+\lambda)^{2}-4(1-\lambda)^{2}\right]^{2}}\right\} e_{r}
$$

where $A$ is the Hamaker constant. $R_{i}$ and $R_{j}$ are the radiuses of two magnetic particles respectively.

$$
\begin{gathered}
A=\left(A_{1}{ }^{1 / 2}-A_{2}{ }^{1 / 2}\right)^{2}, \\
S=\frac{2\left(h+R_{i}+R_{j}\right)}{R_{i}+R_{j}}, \\
\lambda=\frac{R_{i}}{R_{j}}
\end{gathered}
$$

where $A_{1}$ and $A_{2}$ are the Hamaker constants of magnetic particles and carrier liquid, respectively. 


\subsubsection{Repulsive Force}

The repulsive force plays a vital role in preventing the particles from overlapping and aggregating. The repulsive force can be derived by following formula [29]:

$$
F_{i}^{R}=\sum_{j \neq i} F_{0} \exp \left[-B\left(\frac{r_{i j}}{2 R}-1\right)\right] \widehat{r}_{i j}
$$

where $B$ decides the variation rate of repulsive force along with the change of the deformation of magnetic particles.

The parameter $F_{0}$ is the repulsive force when two magnetic particles come into contact in the direction of the exterior magnetic field. Through the balance of magnetic force and repulsive force $F_{i}^{r}=-F_{i}^{m}$, and $F_{0}$ can be calculated as follows:

$$
F_{0}=\frac{3 u_{0} m^{2}}{2 \pi(2 R)^{4}}
$$

\subsubsection{The Sum of Gravitational Force and Buoyant Force}

The sum of gravitational force and buoyant force can be calculated as follows:

$$
F_{i}^{H}=\frac{4 \pi R^{3}}{3} \Delta \rho g
$$

where $\Delta \rho$ is the mass density difference between the suspended magnetic particles and carrier liquid, and $g$ is the acceleration of gravitation.

The resultant force $F^{\sigma}$ acting on magnetic particles is the vector sum of above internal and external forces, and it can be expressed as

$$
F_{i}^{\sigma}=F_{i}^{B}+F_{i}^{V}+F_{i}^{M}+F_{i}^{W}+F_{i}^{R}+F_{i}^{H}
$$

\subsection{Two-Component Lattice Boltzmann Model}

The magnetorheological fluids composed of suspended magnetic particles and carrier liquid is a two-component system. In this paper, the well-known D2Q9 model is employed to simulate magnetorheological fluids. The nine possible velocities at each node for the magnetic particles can be expressed as follows:

$$
e_{i}= \begin{cases}0 & i=0 \\ c\left(\cos \left[\frac{(i-1) \pi}{2}\right], \sin \left[\frac{(i-1) \pi}{2}\right]\right) & i=1,2,3,4 \\ \sqrt{2} c\left(\cos \left[\frac{(i-1) \pi}{2}\right], \sin \left[\frac{(i-1) \pi}{2}\right]\right) & i=5,6,7,8\end{cases}
$$

where $c=\delta_{x} / \delta_{t}$ is the lattice velocity, $\delta_{x}$ and $\delta_{t}$ are the space step and time step, respectively. 
The Lattice Boltzmann evolution equation can be proposed for each component $\sigma$ in the magnetorheological fluids as follows:

$$
f_{i}^{\sigma}\left(x+e_{i} \delta_{t}, t+\delta_{t}\right)-f_{i}^{\sigma}(x, t)=-\frac{1}{\tau^{\sigma}}\left[f_{i}^{\sigma}(x, t)-f_{i}^{\sigma, e q}(x, t)\right] \quad i=0,1, \ldots, 8,
$$

where $\sigma=1,2$ is the index of two phase particles of magnetorheological fluids. $f_{i}^{\sigma}(x, t)$ is the distribution function for the particles of component $\sigma$ which have microscopic velocity $e_{i}$ at lattice site $x$ on time $t . \tau^{\sigma}$ is the single-relaxation time of each component which controls the rate approaching equilibrium. $f_{i}^{\sigma, e q}$ is the equilibrium distribution function of the component $\sigma$.

The equilibrium distribution function can be expressed by a discretization of the Maxwell-Boltzmann equilibrium distribution shown as follows:

$$
f_{i}^{\sigma, e q}=\rho^{\sigma} \omega_{i}\left[1+\frac{3 e_{i} \cdot u^{\sigma, e q}}{c^{2}}+\frac{9\left(e_{i} \cdot u^{\sigma, e q}\right)^{2}}{2 c^{4}}-\frac{3 u^{\sigma, e q 2}}{2 c^{2}}\right]
$$

where $\omega_{i}$ is a weighting factor which depends on Lattice Boltzmann model. In the D2Q9 model, $\omega_{i}=4 / 9(i=0), \omega_{i}=1 / 9(i=1,2,3,4), \omega_{i}=1 / 36(i=5,678)$, and $u^{\sigma, e q}$ is the local equilibrium velocity of each component $\sigma$.

The macroscopic density $\rho^{\sigma}$ and velocity $u^{\sigma}$ of each component $\sigma$ can be calculated with the following relationships:

$$
\rho^{\sigma}=\sum_{i} f_{i}^{\sigma}, \quad u^{\sigma}=\frac{1}{\rho^{\sigma}} \sum_{i} f_{i}^{\sigma} e_{i}
$$

Thus, the magnetorheological fluids macroscopic density $\rho$ and velocity $u$ can be presented as follows:

$$
\rho=\sum_{\sigma} \rho^{\sigma}, \quad u=\frac{\sum_{\sigma} \rho^{\sigma} u^{\sigma}}{\sum_{\sigma} \rho^{\sigma}}
$$

In the absence of any additional force acting on the each component particles, the equilibrium velocity of each component is assumed to be equal to the mixed velocity $u^{\prime}$ of the two-component magnetorheological fluids, and it follows the requirement that the total momentum must be conserved at each collision. The local equilibrium velocity can be defined as follows [30]:

$$
u^{\prime}=\frac{\sum_{\sigma}\left(\rho^{\sigma} u^{\sigma} / \tau_{\rho}^{\sigma}\right)}{\sum_{\sigma}\left(\rho^{\sigma} / \tau_{\rho}^{\sigma}\right)} .
$$

Under the effect of an external magnetic field, magnetic particles would be affected by the various internal and external forces. So it is necessary to revise the local equilibrium speed with taking into account the factor of all forces. In the presence of external and interparticle 
forces vector, each component particle gains a momentum variation at each site, and the new equilibrium velocity can be proposed as follows:

$$
u^{\sigma, e q}=u^{\prime}+\frac{F^{\sigma} \tau_{\rho}^{\sigma} \Delta t}{\rho^{\sigma}}
$$

\section{The Flowchart of LBM with Double Meshes}

The detailed process for the simulation of magnetorheological fluids based on LBM with double meshes can be designed as follows:

(1) The initial density $\rho^{(0)}$ and velocity $u^{(0)}$ are derived according to concrete problems.

(2) According to $\rho^{(0)}$ and $u^{(0)}$, calculate the initial distribution function $f_{i}^{(0)}$ of suspended magnetic particles by (3.13).

(3) On the fine mesh, the distribution function $f_{f}^{(m)}$ is obtained after iterating for $m$ times by (3.12).

(4) Calculate the density $\rho_{f}^{(m)}$ and velocity $u_{f}^{(m)}$ of magnetic particles by (3.14).

(5) Transfer $\rho_{f}^{(m)}$ and $u_{f}^{(m)}$ from the fine mesh to coarse mesh by the following formulas:

$$
\rho_{c}=I_{f}^{c} \rho_{f}^{(m)}, \quad u_{c}=I_{f}^{c} u_{f}^{(m)}
$$

where $I_{f}^{c}$ is the prolongation operator.

(6) According to $\rho_{c}$ and $u_{c}$, calculate the distribution function $f_{i}$ by (3.13).

(7) On the coarse mesh, the distribution function $f_{c}^{(n)}$ is obtained after iterating for $n$ times by (3.12).

(8) Calculate the density $\rho_{c}^{(n)}$ and velocity $u_{c}^{(n)}$ of magnetic particles by (3.14).

(9) Transfer $\rho_{c}^{(n)}$ and $u_{c}^{(n)}$ from the coarse mesh to fine mesh by the following formulas:

$$
\rho_{f}=I_{c}^{f} \rho_{c}^{(n)}, \quad u_{f}=I_{c}^{f} u_{c}^{(n)}
$$

where $I_{c}^{f}$ is the restriction operator.

(10) Set $\rho_{f}$ to $\rho^{(0)}$ and $u_{f}$ to $u^{(0)}$ and repeat (2) (10) steps until the computation is convergent.

It is very important for the simulation of magnetorheological fluids to couple the variables and parameters from different scale regions. As shown in the fifth and ninth steps, the prolongation operator and restriction operator are adopted to transfer the variables and parameters between the fine and coarse meshes. So it requires that the prolongation operator and restriction operator should have high accuracy. Combined with the distribution of the double meshes shown as Figure 1, the prolongation operator and restriction operator can be calculated as follows. 
The density $\rho_{c}$ and velocity $u_{c}$ of magnetorheological fluids on the coarse mesh are obtained by means of integration of $\rho_{c}$ and $u_{c}$ from the fine grids, that is $\rho_{c}=I_{f}^{c} \rho_{f}^{(m)}, u_{c}=$ $I_{f}^{c} u_{f}^{(m)}$. The linear interpolation is shown as follows in the two-dimensional simulation:

$$
\begin{gathered}
\rho\left[i_{c}, j_{c}\right]=\frac{1}{16}\left(\rho\left[i_{f}-1, j_{f}+1\right]+2 \rho\left[i_{f}, j_{f}+1\right]+\rho\left[i_{f}+1, j_{f}+1\right]+2 \rho\left[i_{f}-1, j_{f}\right]\right. \\
\left.+4 \rho\left[i_{f}, j_{f}\right]+2 \rho\left[i_{f}+1, j_{f}\right]+\rho\left[i_{f}-1, j_{f}-1\right]+2 \rho\left[i_{f}, j_{f}-1\right]+\rho\left[i_{f}+1, j_{f}-1\right]\right), \\
u\left[i_{c}, j_{c}\right]=\frac{1}{16}\left(u\left[i_{f}-1, j_{f}+1\right]+2 u\left[i_{f}, j_{f}+1\right]+u\left[i_{f}+1, j_{f}+1\right]+2 u\left[i_{f}-1, j_{f}\right]+4 u\left[i_{f}, j_{f}\right]\right. \\
\left.+2 u\left[i_{f}+1, j_{f}\right]+u\left[i_{f}-1, j_{f}-1\right]+2 u\left[i_{f}, j_{f}-1\right]+u\left[i_{f}+1, j_{f}-1\right]\right) .
\end{gathered}
$$

On the other hand, the density $\rho_{f}$ and velocity $u_{f}$ of magnetorheological fluids on the fine mesh are obtained by means of integration of $\rho_{c}^{(n)}$ and $u_{c}^{(n)}$ from the coarse grids, that is $\rho_{f}=I_{c}^{f} \rho_{c}^{(n)}, u_{f}=I_{c}^{f} u_{c}^{(n)}$. In the two-dimensional simulation, the restriction operator $I_{c}^{f}$ can be calculated as follows:

$$
\begin{gathered}
\rho\left[i_{f}, j_{f}\right]=\rho\left[i_{c}, j_{c}\right], \\
\rho\left[i_{f}+1, j_{f}\right]=\frac{1}{2}\left(\rho\left[i_{c}, j_{c}\right]+\rho\left[i_{c}+1, j_{c}\right]\right), \\
\rho\left[i_{f}, j_{f}+1\right]=\frac{1}{2}\left(\rho\left[i_{c}, j_{c}\right]+\rho\left[i_{c}, j_{c}+1\right]\right), \\
\rho\left[i_{f}+1, j_{f}+1\right]=\frac{1}{4}\left(\rho\left[i_{c}, j_{c}\right]+\rho\left[i_{c}+1, j_{c}\right]+\rho\left[i_{c}, j_{c}+1\right]+\rho\left[i_{c}+1, j_{c}+1\right]\right), \\
u\left[i_{f}, j_{f}\right]=u\left[i_{c}, j_{c}\right], \\
u\left[i_{f}+1, j_{f}\right]=\frac{1}{2}\left(u\left[i_{c}, j_{c}\right]+u\left[i_{c}+1, j_{c}\right]\right), \\
u\left[i_{f}, j_{f}+1\right]=\frac{1}{2}\left(u\left[i_{c}, j_{c}\right]+u\left[i_{c}, j_{c}+1\right]\right), \\
u\left[i_{f}+1, j_{f}+1\right]=\frac{1}{4}\left(u\left[i_{c}, j_{c}\right]+u\left[i_{c}+1, j_{c}\right]+u\left[i_{c}, j_{c}+1\right]+u\left[i_{c}+1, j_{c}+1\right]\right) .
\end{gathered}
$$

During the simulation of magnetorheological fluids in an external magnetic field, the two-component Lattice Boltzmann method with double meshes can exert the merits of LBM and double mesh method. Thus, it is useful to improve computational efficiency and make the calculation converged easily.

\section{Simulation and Discussion}

In this section, the two-component Lattice Boltzmann method with double meshes is applied to simulate magnetorheological fluids in the different strength magnetic fields. In order to 
Table 1: The parameters of magnetorheological fluids.

\begin{tabular}{lcccc}
\hline Parameter & $R / \mathrm{um}$ & $\chi$ & $\eta / \mathrm{Pa} \cdot \mathrm{s}$ & $A \times 10^{20} / \mathrm{J}$ \\
\hline Value & 10 & 3.2 & 0.01 & 22.3 \\
Parameter & $u_{0} \times 10^{7} /\left(\mathrm{H} \cdot \mathrm{m}^{-1}\right)$ & $\rho_{p} \times 10^{-3} /\left(\mathrm{kg} \cdot \mathrm{m}^{-3}\right)$ & $\rho_{f} \times 10^{-3} /\left(\mathrm{kg} \cdot \mathrm{m}^{-3}\right)$ & \\
Value & $4 \pi$ & 0.89 & 7.8 & \\
\hline
\end{tabular}

verify the validity of two-component Lattice Boltzmann model with double meshes for the simulation of magnetorheological fluids, a physics experiment is carried out. The simulation results are contrasted with the physics experiment results and the computational efficiency of the proposed approach is compared with other methods.

The above forces decrease quickly along with the increasing of distance between two particles. So during the simulation of magnetorheological fluids, it is just needed to calculate the forces from other particles at adjacent nodes to reduce the simulation time. The parameters of magnetorheological fluids used in simulation are shown as in Table 1. The volume fraction of magnetorheological fluids used in our simulation is $10 \%$. The fluids are confined between two parallel plates with the distance of $L=80 \mathrm{~mm}$ and the fluid temperature is $293 \mathrm{~K}$. The initial velocity is assumed to be zero. The spatial steps and the time steps are $\delta_{x}=500 \mathrm{um}, \delta_{t}=2 \times 10^{-7} \mathrm{~s}$, respectively. Periodic boundary condition is adopted at the entrance and exit of the channel and bounce-back boundary condition is used at the top and bottom boundary.

Figure 2 is the simulation results for microstructure of magnetorheological fluids in the different strength magnetic fields through the two-component Lattice Boltzmann model with double meshes. The random distribution of magnetic particles in the absence of an external magnetic field is shown as Figure 2(a). Figure 2(b) shows that short clusters are formed sporadically in a low magnetic field $\lambda=1.5 \mathrm{mT}$. For the case of $\lambda=3.0 \mathrm{mT}$, many long thin chainlike clusters are formed along the magnetic field direction as shown in Figure 2(c). In the strong magnetic field $\lambda=6.0 \mathrm{mT}$, the chainlike clusters gradually draw together and form thick chainlike clusters along the strong magnetic field direction as shown in Figure 2(d).

Figure 3 shows the physics experimental results about distributions of magnetic particles on the same condition and the simulation results are in a good accordance with these of experiment values. In other words, the two-component Lattice Boltzmann model with double meshes is feasible and efficient for the simulation of magnetorheological fluids.

A high computational efficiency is crucial to a numerical approach, so it is necessary to investigate the factors which may affect the computational efficiency. In this section, we compare the computational time of three different models in the simulation of magnetorheological fluids at the different volume fraction. The computer used in our paper is Pentium(R) Dual-Core CPU E5700 3.00 GHz.

Figure 4 shows the computational time related with grid number for three different approaches. The grid number of the coarse and fine meshes used in the double meshes method is respectively $n \times n$ and $2 n \times 2 n$. The grid size used in the single-component Lattice Boltzmann model is equal to the coarse grid size used in the double meshes method and the grid size used in the two-component Lattice Boltzmann model is the same as the fine grid size used in the double meshes method.

The comparison results indicate that the single-component Lattice Boltzmann model consumes the minimum computational time. But because it treats the magnetorheological fluids as a mixed single-component fluid and adopts the coarse meshes, so its simulation results are not accurate and cannot meet the requirement of magnetorheological fluids simulation. 


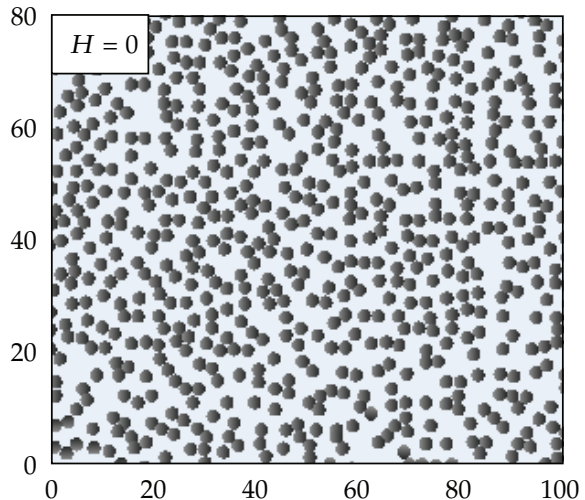

(a)

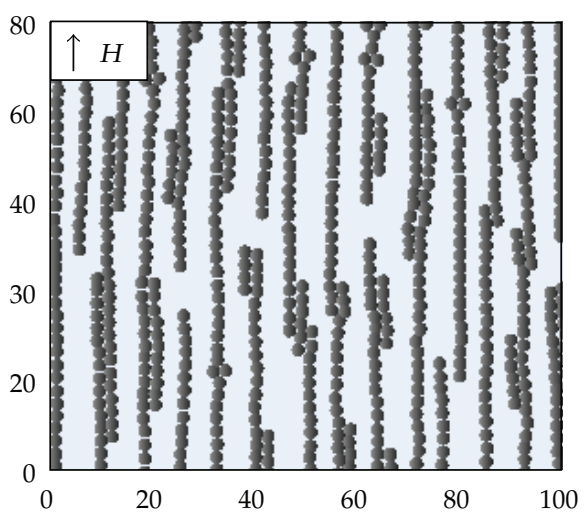

(c)

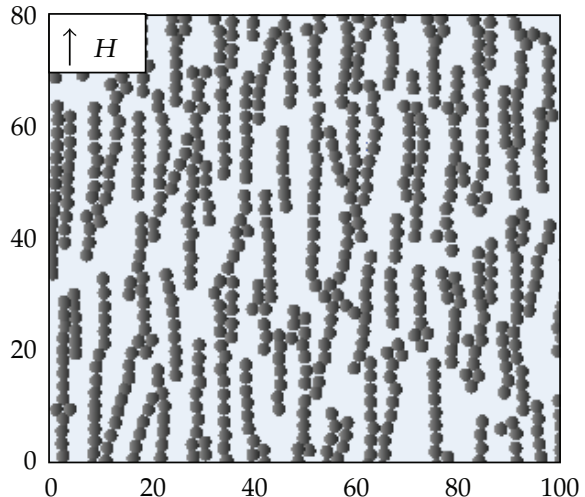

(b)

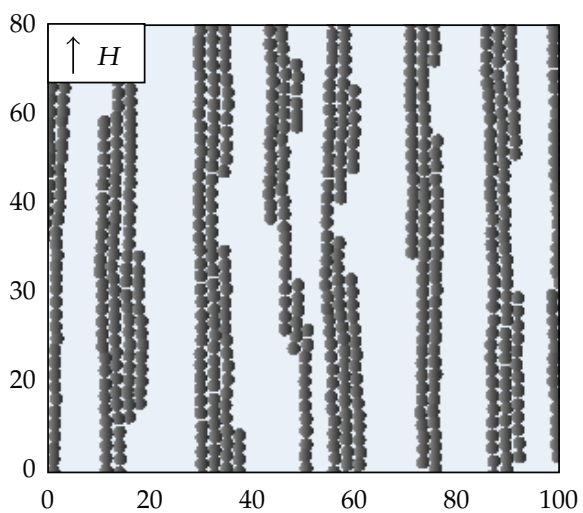

(d)

Figure 2: Influences of magnetic field strength on distributions of particles.

The two-component Lattice Boltzmann model consumes the maximum computational time, and the computational time is reduced obviously through the application of double meshes method.

Figure 5 shows the computational time ratio of two-component LBM with double meshes $\left(T_{2}\right)$ to single-component LBM $\left(T_{1}\right)$ and two-component LBM $\left(T_{3}\right)$ to two-component LBM with double meshes $\left(T_{2}\right)$ as the grid is refined. It can be easy to find the simulation time of magnetorheological fluids with the two-component LBM with double meshes increases faster than the other two approaches along with the grids becoming denser. The reason lies in that the two-component LBM with double meshes takes more time to transfer the values of $u$ and $\rho$ between the coarse and fine meshes with the increasing of grid number, while the other two approaches has not such a process, so the increase of computational efficiency is affected. But the computational efficiency can obviously be improved in the premise of guarantee calculation precision.

\section{Conclusions and Future Work}

It usually takes a lot of time in the simulation of magnetorheological fluids by the uniform mesh algorithm in LBM. In order to improve computational efficiency, the two-component 


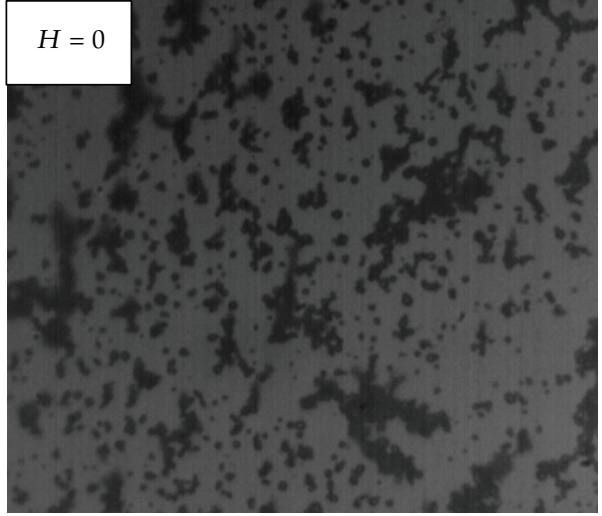

(a)

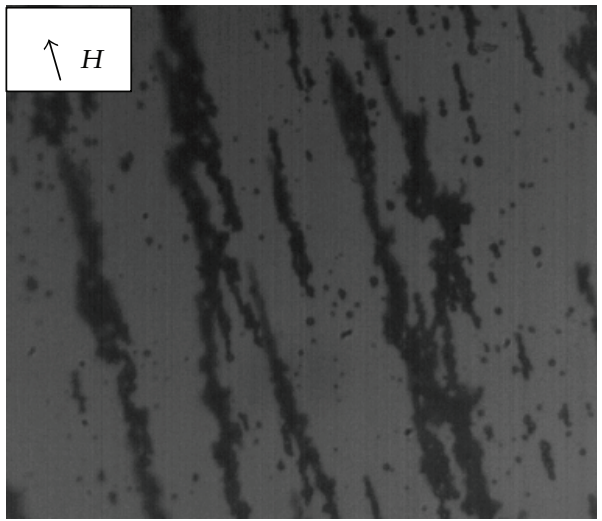

(c)

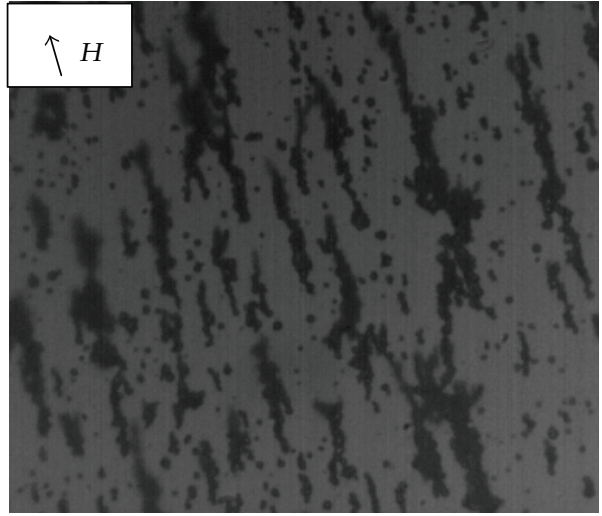

(b)

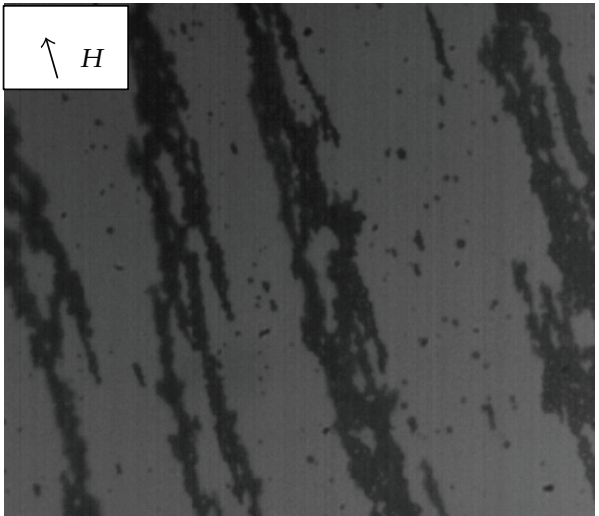

(d)

Figure 3: The physics experiment results in different strength magnetic fields.

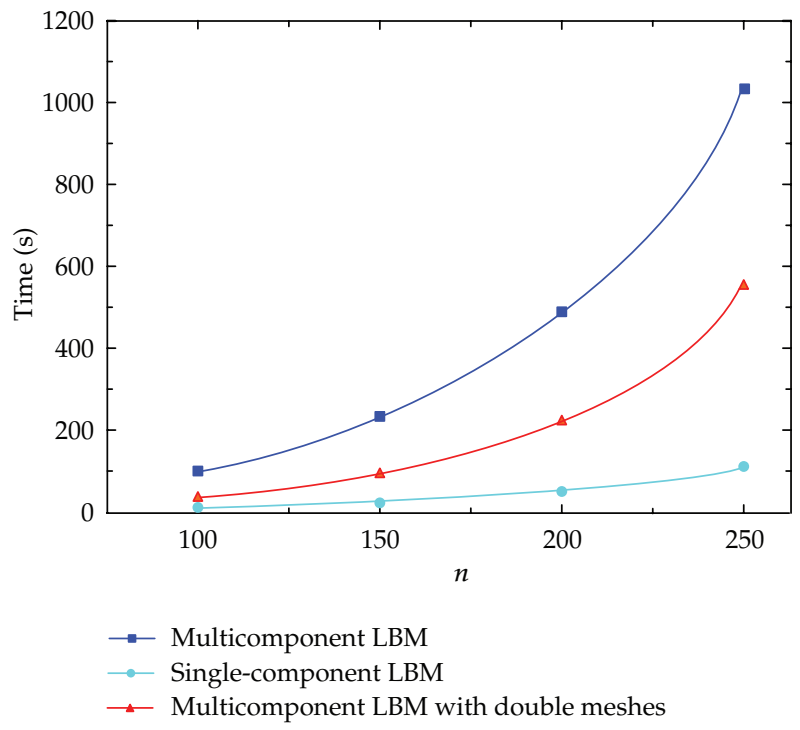

Figure 4: The relation between computation time and grid number. 


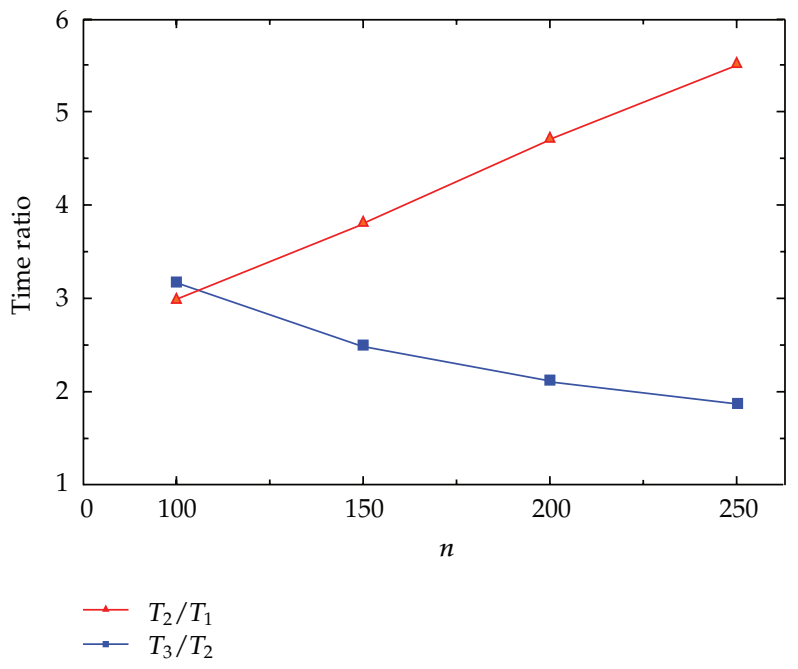

Figure 5: The relation between time ratio and grid number.

Lattice Boltzmann model with double meshes was proposed. During the simulation of magnetorheological fluids in an external magnetic field, the two-component LBM with double meshes can exert the merits of LBM and double mesh method. It makes the high frequency error reduce on the fine mesh and the low frequency error decrease on the coarse mesh. Thus, it is useful to improve computational efficiency and make the calculation converged easily. The comparison between the simulation and physics experiment verifies the validity of two-component Lattice Boltzmann model with double meshes for magnetorheological fluids. The proposed approach has been vital significant to study the rheological characteristics of magnetorheological fluids. However, the simulation results show that the simulation time of magnetorheological fluids increases quickly along with the grids becoming denser. It is due to the factor that the proposed approach would take more time in transferring $u$ and $\rho$ between the fine and coarse meshes. In future studies, the authors plan to investigate the interpolation operator to improve computational efficiency. The application of the proposed approach to the simulation of magnetorheological fluids with a mass volume fraction is also an important research for the authors.

\section{Acknowledgments}

The support of National Natural Science Foundation of China (No. 51005231), National Science Foundation for Post-doctoral Scientists of China (No. 20100471408) and Fundamental Research Funds for the Central Universities (No. 2012QNB14), and the Priority Academic Program Development of Jiangsu Higher Education Institutions in carrying out this research is gratefully acknowledged.

\section{References}

[1] J. Rodríguez-López, L. Elvira Segura, and F. Montero de Espinosa Freijo, “Ultrasonic velocity and amplitude characterization of magnetorheological fluids under magnetic fields," Journal of Magnetism and Magnetic Materials, vol. 324, pp. 222-230, 2012. 
[2] X. He, L. S. Luo, and M. Dembo, "Some progress in the lattice Boltzmann method: reynolds number enhancement in simulations," Physica A, vol. 239, no. 1-3, pp. 276-285, 1997.

[3] C. Shu, X. D. Niu, and Y. T. Chew, "Taylor series expansion and least squares-based lattice Boltzmann method: three-dimensional formulation and its applications," International Journal of Modern Physics C, vol. 14, no. 7, pp. 925-944, 2003.

[4] O. Filippova and D. Hänel, "Grid refinement for lattice-BGK models," Journal of Computational Physics, vol. 147, no. 1, pp. 219-228, 1998.

[5] C. B. Allen, "A multi-block lattice Boltzmann method for viscous fluid flows," International Journal for Numerical Methods in Fluids, vol. 39, no. 2, pp. 99-120, 2002.

[6] J. Tölke, M. Krafczyk, and E. Rank, "A multigrid-solver for the discrete Boltzmann equation," Journal of Statistical Physics, vol. 107, no. 1-2, pp. 573-591, 2002.

[7] J. Sun, "Multiresolution based multigrid methods," Journal of Ningbo University, vol. 21, pp. 538-541, 2008.

[8] X. Peng, Y. Min, T. Ma, and M. Yan, "Two-dimensional Monte Carlo simulations of a suspension comprised of magnetic and nonmagnetic particles in gradient magnetic fields," Journal of Magnetism and Magnetic Materials, vol. 321, no. 19, pp. 3250-3255, 2009.

[9] Y. Xuan, M. Ye, and Q. Li, "Mesoscale simulation of ferrofluid structure," International Journal of Heat and Mass Transfer, vol. 48, no. 12, pp. 2443-2451, 2005.

[10] L. Haitao and P. Xianghe, "Dynamic analysis on ferromagnetic particles of magnetorheological fluids," Journal of Chongqing University, vol. 33, no. 5, pp. 100-113, 2010.

[11] G. Li, Y. Kong, J. Zhang, and J. Zhang, "Simulation and analysis of magnetorheological fluid rheological characteristics based on the theory of magnetic dipoles," Journal of Academy of Armored Force Engineering, vol. 24, pp. 77-81, 2010.

[12] H. V. Ly, F. Reitich, M. R. Jolly, H. T. Banks, and K. Ito, "Simulations of particle dynamics in magnetorheological fluids," Journal of Computational Physics, vol. 155, no. 1, pp. 160-177, 1999.

[13] Z. Zhang, Q. Liu, and F. Huang, "A Method to simulate the MR fluid in ANSYS," Journal of Guizhou University, vol. 28, pp. 110-113, 2011.

[14] L. Zhou, Y. Xuan, and Q. Li, "Multiscale simulation of flow and heat transfer of nanofluid with lattice Boltzmann method," International Journal of Multiphase Flow, vol. 36, no. 5, pp. 364-374, 2010.

[15] B. Nestler, A. Aksi, and M. Selzer, "Combined lattice Boltzmann and phase-field simulations for incompressible fluid flow in porous media," Mathematics and Computers in Simulation, vol. 80, no. 7, pp. 1458-1468, 2010.

[16] S. Gokaltun and G. S. Dulikravich, "Lattice Boltzmann computations of incompressible laminar flow and heat transfer in a constricted channel," Computers \& Mathematics with Applications, vol. 59, no. 7, pp. 2431-2441, 2010.

[17] E. S. Boek and M. Venturoli, "Lattice-Boltzmann studies of fluid flow in porous media with realistic rock geometries," Computers \& Mathematics with Applications, vol. 59, no. 7, pp. 2305-2314, 2010.

[18] V. Heuveline, M. J. Krause, and J. Latt, "Towards a hybrid parallelization of lattice Boltzmann methods," Computers E Mathematics with Applications, vol. 58, no. 5, pp. 1071-1080, 2009.

[19] G. Yan and J. Zhang, "A higher-order moment method of the lattice Boltzmann model for the Korteweg-de Vries equation," Mathematics and Computers in Simulation, vol. 79, no. 5, pp. 1554-1565, 2009.

[20] J. Wang, M. Wang, and Z. Li, "Lattice evolution solution for the nonlinear Poisson-Boltzmann equation in confined domains," Communications in Nonlinear Science and Numerical Simulation, vol. 13, no. 3, pp. 575-583, 2008.

[21] B. Shi and Z. Guo, "Lattice Boltzmann simulation of some nonlinear convection-diffusion equations," Computers \& Mathematics with Applications, vol. 61, no. 12, pp. 3443-3452, 2011.

[22] Y. Duan, R. Liu, and Y. Jiang, "Lattice Boltzmann model for the modified Burgers' equation," Applied Mathematics and Computation, vol. 202, no. 2, pp. 489-497, 2008.

[23] T. Hyakutake, T. Matsumoto, and S. Yanase, "Lattice Boltzmann simulation of blood cell behavior at microvascular bifurcations," Mathematics and Computers in Simulation, vol. 72, no. 2-6, pp. 134-140, 2006.

[24] X. Wang, L. Suo, and D. Liu, "Lattice Boltzmann method with double meshes," Journal of Hydrodynamics, vol. 1, pp. 90-96, 2003.

[25] M. M. Kim and A. L. Zydney, "Effect of electrostatic, hydrodynamic, and Brownian forces on particle trajectories and sieving in normal flow filtration," Journal of Colloid and Interface Science, vol. 269, no. 2, pp. 425-431, 2004. 
[26] S. Fang and P. Zhang, "Simulation of the structure and the dynamics of the particles of MR fluids in rotating magnetic fields," Chinese Journal of Chemical Physics, vol. 14, no. 5, pp. 565-566, 2001.

[27] J. He and G. Miao, "The interaction between two magnetic dipoles," Journal of Nanchang University, vol. 18, pp. 96-98, 1996.

[28] A. D. Ebner, J. A. Ritter, and H. J. Ploehn, "Magnetic hetero-flocculation of paramagnetic colloidal particles," Journal of Colloid and Interface Science, vol. 225, no. 1, pp. 39-46, 2000.

[29] S. Melle, O. G. Calderón, G. G. Fuller, and M. A. Rubio, "Polarizable particle aggregation under rotating magnetic fields using scattering dichroism," Journal of Colloid and Interface Science, vol. 247, no. 1, pp. 200-209, 2002.

[30] Y. Xuan, Q. Li, and Z. Yao, "Lattice Botzmann simulation for nanofluids," Science in China Technological Sciences, vol. 34, pp. 280-287, 2004. 


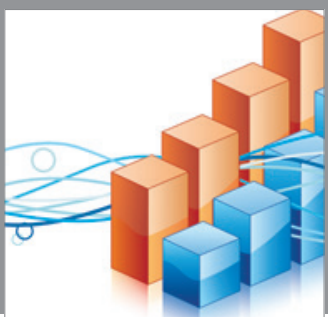

Advances in

Operations Research

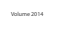

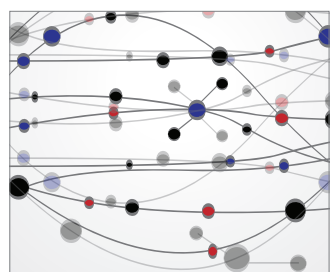

\section{The Scientific} World Journal
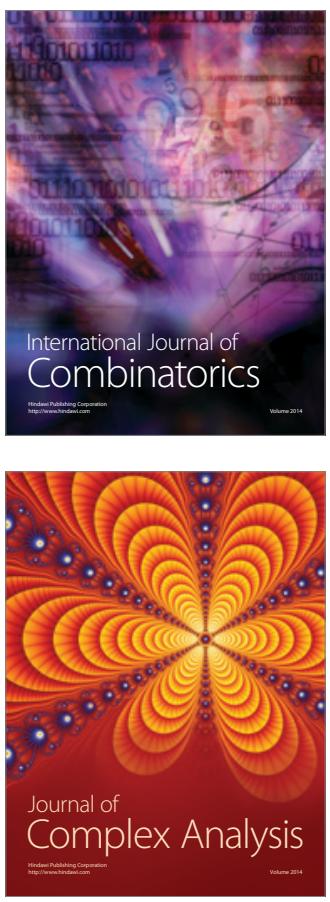

International Journal of

Mathematics and

Mathematical

Sciences
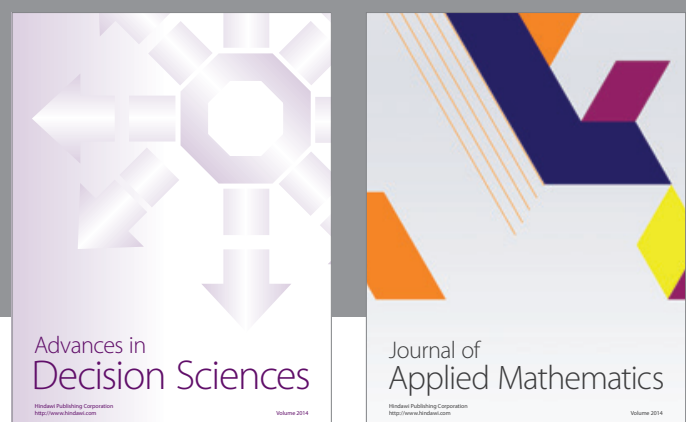

Journal of

Applied Mathematics
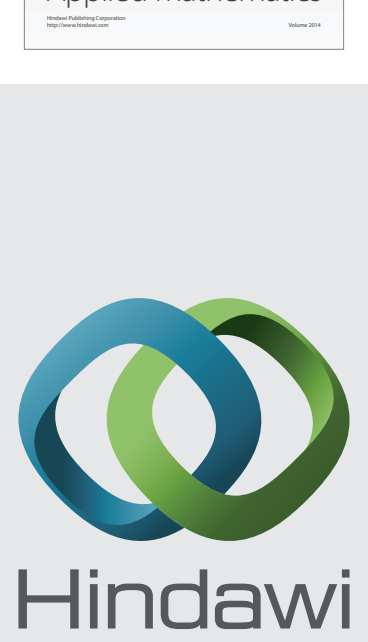

Submit your manuscripts at http://www.hindawi.com
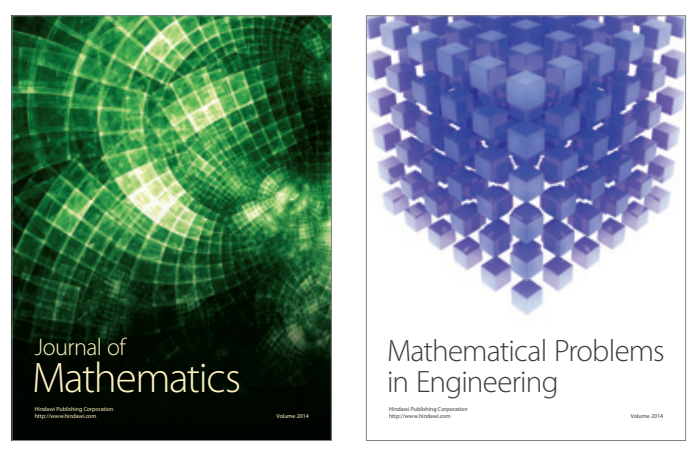

Mathematical Problems in Engineering
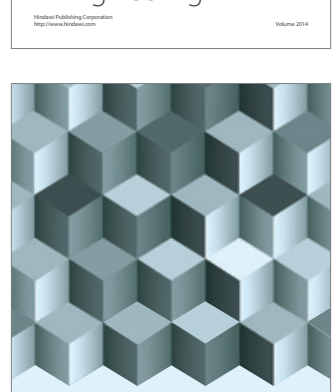

Journal of

Function Spaces
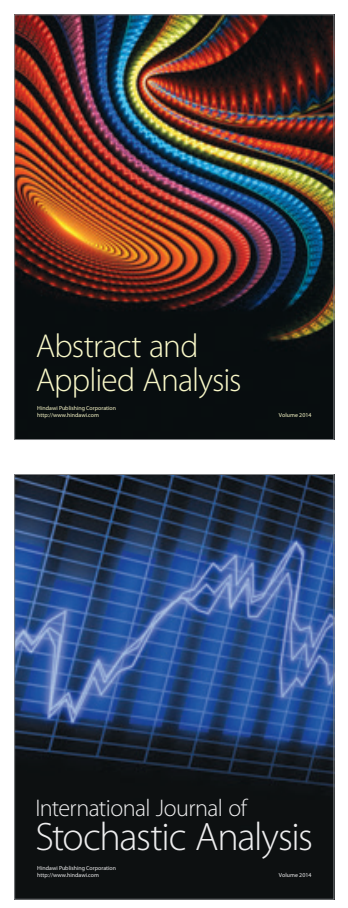

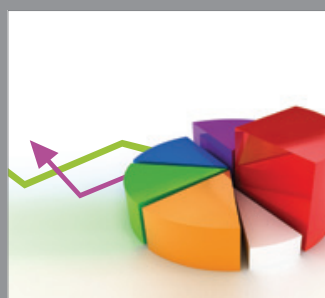

ournal of

Probability and Statistics

Promensencen
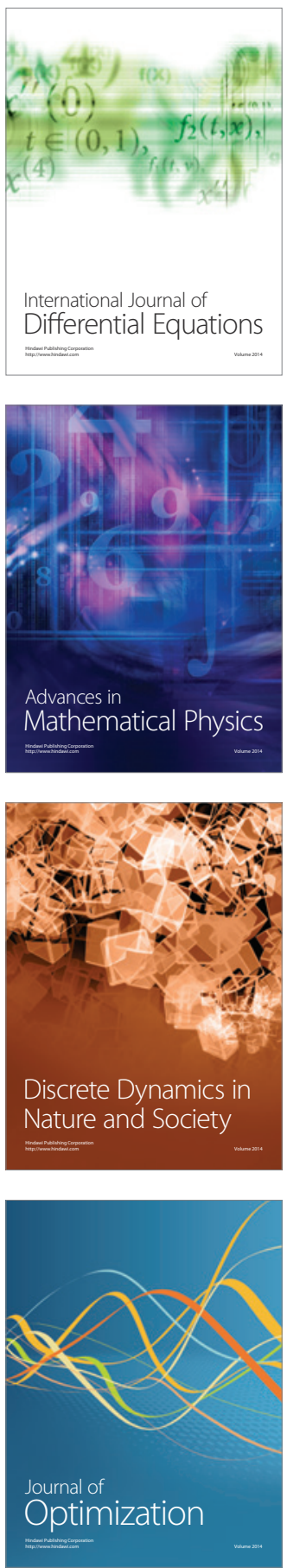